\title{
Image Data Denoising using Center Pixel Weights in Non-Local Means and Smart Patch-based, Modern Machine Learning Method using Higher Order Singular Value Decomposition: A Review
}

\author{
Jeetesh Kumar Rajak \\ M.Tech student \\ Dept. of Electronics \& communication Mittal \\ Institute of Technology Bhopal (MP)
}

\author{
Achint Chugh \\ Assistant Professor \\ Dept. of Electronics \& communication Mittal \\ Institute of Technology Bhopal (MP)
}

\begin{abstract}
In this work, there is a comparison related to image denoising techniques between center pixel weights (CPW) in Non-Local Means (NLM) and smart patch-based, modern technique using the higher order singular value decomposition (HOSVD). The HOSVD technique simply compose in a cluster, alike Patches of noisy image in 3D heap, work out HOSVD factors of this heap, handles these factors by stiff thresholding, and turn upside down the HOSVD transmute to yield the final resultant image. Whereas (NLM) and its variants have proven to be effective and robust in many image denoising tasks. It is experimentally demonstrating approximately 12 percent improved PSNR characteristics of HOSVD technique on gray scale images. The HOSVD process yields state-of-the-art outcomes on gray images, than the center pixel weights (CPW) in NLM image data denoising process at moderately great noise stages.
\end{abstract}

\section{Keywords}

Image data denoising, singular value decomposition (SVD), HOSVD, patch Basis similarity

\section{INTRODUCTION}

The image noise removable has a very prosperous history. An overabundance of distinct techniques has been offered, certain techniques we will analysis later. In present times, the transform-based methods mainly in combination through machine learning popularity have increased and achievements obtained on the basis of performance obtained. In this paper we have an uncomplicated graceful and useful algorithm that contributes to the impression of study a point wise shifting in term of transform corrupted image pixels with applying the nonlocal self-similarity of image. An effective explanation follows. Assumed an image $I_{n}$ which is the corrupted form of a basic clear image $\mathrm{I}$, our objective is to improve a measurement of $\mathrm{I}_{\text {fromI }}$. We consider a zero mean i.i.d. Gaussian distribution of unchanged, common standard deviation $\sigma($ i. e, $\mathcal{N}(0, \sigma))$ like a noise model. The following steps are using in the noise removable algorithm:

1. A heap consist of identical image patches is designed for all pixel and as a static spot size[15].

2. For each stack, higher order singular value decomposition (HOSVD) bases (3D for gray scale and $4 \mathrm{D}$ for color) are derived [15].

3. All heaps is planned on top of the bases and coefficients through values under a hard threshold are reduced to get a bunch of hypotheses [15].
4. The patches are reconstructed in image area and the bunch of hypotheses at all pixel averaged to achieve a noised free image [15].

The just free parameter is the patch size. This HOSVD based image noise removable algorithm get near to the advanced performance. We express example outcomes of our method on a gray scale depraved via noise from $\mathcal{N}(0,20)$ and a color version of the identical image in $\mathcal{N}(0,20)$ noise on $\mathrm{R}, \mathrm{G}$, and B.

Image noise generally occurs into the image transmission, quantization, acquisition and a many different processing steps. A digital image polluted through computer visualization tasks like classification, tracking, recognition, etc. and can affect several progressive image processing and noises leads to visible loss in image quality. The importance of image noise removable is therefore commonly accepted. Conventional image noise removable methods like as moving average filters, wavelet filter and Wiener filters banks are powerfully related to typical filtering[1].These filter created image noise removable methods are usually of low difficulty and can be simply achieved. But their performance is not all the time acceptable. By the improved computational capacity of modern processors, various advanced noise removable methods are now possible. With these method the Non-Local Means (NLM) technique [1], [2] has involved important awareness in current years. The Non-Local Means (NLM) remove the noise in an image pixel as the weighted sum of its corrupted neighbours, where every weight replicates the similarity among the local patch centred of the pixel to be noise removed and patch centred of the neighbour pixel. NLM familiarizes the noise removable process for every pixel and thus out performs conventional methods [1]. Other technique noise removable approaches consist of the LJSCPW method [3], which is equated to Non-Local Means (NLM) method in [4], [5].

The significance of center pixel weights in Non-Local Means has long been known [1], [6], and several weights have been designed [6]. But, the techniques not consider all conditions of CPW problem and they are non-ideal. Thus, new center pixel weights require to be created. In this work, we examine the CPW issue in Non-Local Means (NLM) and propose new results founded on the James-Stein estimator [10]. In this work issystematized study the Non-Local Means also connected work on CPWs; the new formulation of the CPW problem and the new James-Stein type CPWs. 


\section{THE JAMES-STEIN CENTRE PIXEL WEIGHT [16]}

A significant solution in reduction estimators is the JamesStein estimator [10]. It states that for an unknown parameter vector and observations of with the relation,

$$
\mathrm{b} \sim \mathrm{N}\left(\mathrm{a}, \sigma^{2} \mathrm{I}\right)
$$

There follows a James-Stein estimator that reduces near an arbitrary vector in this equation,

$$
\hat{a}^{J S}=c+q(b-c)=(1-q) c+q b
$$

With the coefficient q of form

$$
\mathrm{q}=1-(\mathrm{m}-2) \sigma^{2} /\|\mathrm{b}-\mathrm{c}\|^{2}
$$

The James-Stein estimator is a classic solution that reduces the risk of estimation in terms of the errorE[ $\left[\|\mathrm{a}-\hat{a}\|^{2}\right]$ where $\|\cdot\|$ represents the $L^{2}$ norm. In the setting of NLM-CPW problem, the James-Stein (JS) based CPW [16] has the weight of form

$$
\mathrm{p}^{\mathrm{JS}}=1-(\mathrm{m}-2) \sigma^{2} /\|\mathrm{y}-\hat{\mathrm{z}}\|^{2}
$$

Where $m=|\mathbb{I}|$ is show the number of pixels into image and the equivalent new estimator is

$$
\hat{\mathrm{X}}^{\mathrm{JS}}=\left(1-\mathrm{p}^{\mathrm{JS}}\right) \hat{\mathrm{z}}+\mathrm{p}^{\mathrm{JS}} \mathrm{y}
$$

To lower the computational cost of the LJSCPW in NLM, we construct the integral image [7] $\mathrm{R}$ with 2 operations/pixel for the pixel-wise mean square error between $\hat{z}$ and $y$. Each pixel is the summation of form

$$
\mathrm{R}_{\mathrm{l}}=\sum_{\mathrm{i}=\left\{\left(\mathrm{i}_{1}, \mathrm{i}_{2}\right) \mid \mathrm{i}_{1} \in\left[1, \mathrm{i}_{1}\right], \mathrm{i}_{1} \in\left[1, \mathrm{i}_{2}\right]\right\}}\left(\mathrm{y}_{\mathrm{i}}-\hat{\mathrm{z}}_{\mathrm{i}}\right)^{2}(6)
$$

This integral image $\mathrm{R}$ then allows computation of $\| y b_{1}-$ zbl2for an arbitrary rectangular B with 3operations/pixel. Considering the extra 4 operations/pixel to compute $p_{l}^{\text {LJS }}$ and the 4 operations/pixel to compute $\hat{x}_{1}^{L J S}$, in total the proposed LJSCPW requires additional 13 operations /pixel. Compared to the NLM complexity of $|\mathbb{P}| \times|\mathbb{S}|$ operations/pixel this shrinkage cost is negligible.

In processing, one may see. $p_{l}^{\mathrm{LJS}}<0$ For some pixels, which conflicts with our assumption that $p_{l}^{\mathrm{LJS}} \in[0,1]$.This occurs when $\hat{z} r_{1}$ is a slightly denoised version of $\mathrm{yr}_{1}$. It is reasonable to use $\widehat{z}_{l}$ rather than $y_{1}$ implyingp $p_{l}^{L J S}=0$ therefore we use the positive part of $\mathrm{p}_{\mathrm{l}}^{\mathrm{LJS}}$ in (21), namely

$$
\mathrm{p}_{\mathrm{l}}^{\mathrm{LJS}}=\max \left(1-(|\mathbb{B}|-2) \sigma^{2} /\left\|\mathrm{y} \mathrm{b}_{\mathrm{l}}-\mathrm{z} \widehat{\mathrm{b}_{\mathrm{l}}}\right\|^{2}, 0\right)
$$

Since $p_{l}^{\text {LJS }}=1$ would indicate the raw pixel is used, it may prove useful in some applications to limit $p_{l}^{\text {LJS }}$ to a user-defined value less than unity. In this letter, however, we allow the shrinkage operator to operate over the full range.

\section{HIGHER ORDER SINGULAR VALUE DECOMPOSITIO [15]}

In the NL-SVD procedure let consider original uncontaminated patch is $Q_{\text {ref }}$ and its reference patch $P_{\text {ref }}$ in the corrupted image. Now, suppose a situation where every $\mathrm{K}$ patches $\left\{P_{i}\right\}$ which is corrupted forms of $Q_{\text {ref }}$. In this situation, we notice that $\lim _{k \rightarrow \infty} \sum_{i=1}^{k} p_{i} p_{i}^{T}=Q_{r e f} Q_{r e f}^{T}+\sigma^{2} I$. as a result for greater $\mathrm{K}$, we have an opportunity of being capable to approximate the singular value decomposition bases of $Q_{\text {ref }}$ and thus access the revelation estimator [15]. But, such a condition is not probable in most natural images, and the patches that succeed as alike will typically not be replica of $Q_{\text {ref }}$ modulo noise Therefore, we accept the following standard: If a bunch of patches are parallel to one another in the corrupted image, The noise removable should take this statement in the account and not noise removable them individually. With this is in mind, we cluster together identical patches and characterize them in the form of a 3D stack. The key point is that the cleaning is accomplished not only through the length and range of all singular (2D) patch, however similarly in the 3rd dimension so as to permit for similarity between intensity values at equivalent pixels of the dissimilar patches. The joint cleaning of several patches has been employed previous in the LJSCPW algorithm but with unchanging bases. On the other hand, in this work, we using this proposal to examine spatially adaptive bases. The advantage of our HOSVD methodology over NL-SVD, for de-noising a section of the boat image the application of coefficient thresholding for flattening of the structurally identical patches of size 64 by the NL-SVD and HOSVD transforms, respectively while the end two rows illustrate the cleaned patches after the averaging processes [15]. These figures disclose that HOSVD conserves the better textures on the tablecloth surface much finer than NL-SVD, which virtually removes those textures. We have also experimentally confirmed the importance of building the stack from similar patches: Randomly generated stacks create transforms that yield unclear and images.

\section{HOSVD FOR DENOISING}

We generate a stack of $K-1$ parallel patches of given a $p \times p$ reference patch $P_{\text {ref }}$ in the corrupted image $I_{n}$, Here, likeness is demarcated and hence the significance of $\mathrm{K}$ diverges from one pixel to another pixel. Now we indicate the stack as $\mathrm{Z} \in \mathrm{R}^{\mathrm{p} \times \mathrm{p} \times \mathrm{k}}$. The HOSVD equation of given stack is,

$$
\mathrm{z}=\mathrm{S} \times \times_{1} \mathrm{U}^{(1)} \times_{2} \mathrm{U}^{(2)} \times_{3} \mathrm{U}^{(3)}
$$

Where $\mathrm{U}^{(1)} \in \mathrm{R}^{\mathrm{p} \times \mathrm{p}}, \mathrm{U}^{(1)} \in \mathrm{R}^{\mathrm{p} \times \mathrm{p}}$, and $\mathrm{U}^{(3)} \in \mathrm{R}^{\mathrm{k} \times \mathrm{k}}$ are orthonormal matrices, and $\mathrm{S}$ is represent as $3 \mathrm{D}$ coefficient array of size $\mathrm{p} \times \mathrm{p} \times \mathrm{k}$ Here, the symbol $\times_{\mathrm{n}}$ given as nth mode tensor product well-defined in [12].The orthonormal matrices $U^{(1)}, U^{(2)}$ and $U^{(3)}$ are, in repetition, calculated from

the Singular Value Decomposition of the unfolding $\mathrm{Z}_{(1)}, \mathrm{Z}_{(2)}$ $\operatorname{andZ}_{(3)}$ respectively [12]. The exact Equation are the form,

$$
\mathrm{Z}_{(\mathrm{k})}=\mathrm{U}^{(\mathrm{k})} \cdot \mathrm{S}_{(\mathrm{k})} \cdot\left(\mathrm{U}^{\bmod (\mathrm{k}+1,3)} \otimes \mathrm{U}^{\bmod (\mathrm{k}+2,3)}\right)^{\mathrm{T}}
$$

Where $1 \leq \mathrm{K} \leq 3$ (which are corresponding representations for the HOSVD). For $\mathrm{k} \times \mathrm{k}$ matrices is $\mathrm{O}$ (K3). For computational speed, we impose the constraint that $\mathrm{K} \leq$ 30 The patches of $\mathrm{Z}$ are at that time estimated onto the HOSVD transform. The limitation for thresholding the transform coefficients are selected to be $\sigma \sqrt{2 \log ^{2} \mathrm{k}}$ again. 


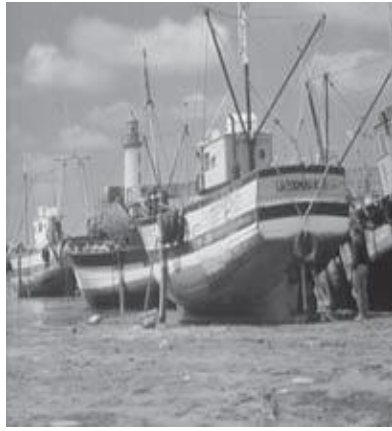

Original image

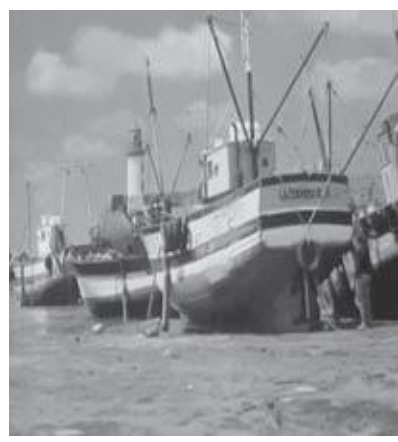

NLM with LJSCPW

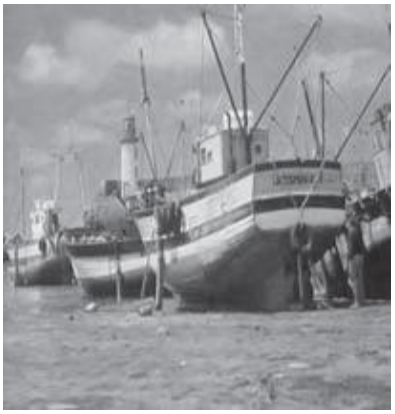

HOSVD 2 image

Fig.1. Left to right: Original image, noisy image under N $(0,40)$, outputs of NLM with LJSCPW (PSNR:

26.339), 4D-HOSVD (PSNR: 26.192), and 4D-

HOSVD2 (PSNR: 26.7). Zoom into pdf file for better view.

The stack of $\mathrm{Z}$ is at that time reassembled after inverting the transform [13], thereby filtering the every different patches into the collective and not take just reference patch. This gives extra flattening on every patches, which was essential because of the upper limit of $\mathrm{K} \leq 30$, dissimilar the case using NL-SVD, We as well enhance the HOSVD denoising through a Wiener, let $\mathrm{Z}$ be a stack of parallel patches of the HOSVD cleaned image and $\mathrm{Zn}$ be the equivalent stack of the blaring image. Let the coefficients of $\widehat{Z}$ and $\mathrm{Zn}$ on the HOSVD bases of $\mathrm{Z}$ be indicated as cand $\mathrm{c}_{\mathrm{n}}$, individually. Formerly, the

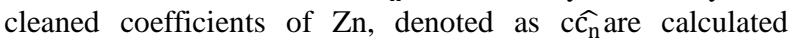
followed through the typical transform inversion and averaging:

$$
\widehat{c}_{\mathrm{n}}=\frac{\mathrm{c}_{\mathrm{n}} \widehat{\mathrm{c}}^{2}}{\hat{\mathrm{c}}^{2}+\sigma^{2}}
$$

We call this second stage HOSVD.

\section{EXPERIMENTAL RESULTS}

All following simulations are done under the MATLAB r2010a environment with Intel Core CPU at 2.0 GHz. We display PSNR and SSIM values for denoising every images under independent $\mathrm{N}(0, \sigma)$ noise on all channel (where $\sigma \in$ $\{30,40,50\})$, in Tables 1 . Just as done for gray scale images, for all values, the noisy images were generated by adding Gaussian noise to the original image and converting the result to an image file ([0-255] range). We compare our results using HOSVD on 4D stacks (termed "4DHOSVD," with the different channels representing the fourth dimension) with NL Means and the color version of LJSCPW in YCbCr color space. We also implemented a Wiener filter step on top of 4D-HOSVD, which we term as 4D-HOSVD2. We also implemented the following variant of our HOSVD technique: We learned a decorrelated color space from the noisy R, G, B values by principal components analysis and then applied the HOSVD denoising algorithm for gray scale images independently on the three resulting PCA-transformed channels.

Table 1 PSNR Results for Color Image (and SSIM Value on Gray scale Versions) Corrupted by $\mathbf{N}(0,50)$

\begin{tabular}{cccc}
\hline Image & $\begin{array}{c}\text { NLM with } \\
\text { LJSCPW }\end{array}$ & HOSVD1 & HOSVD2 \\
\hline 1 & $24.03,0.12$ & $24.995,0.646$ & $25.327,0.671$ \\
2 & $25.13,0.05$ & $28.278,0.711$ & $28.426,0.718$ \\
3 & $25.32,0.08$ & $29.654,0.803$ & $29.551,0.811$ \\
4 & $26.23,0.10$ & $28.654,0.736$ & $28.900,0.745$ \\
\hline
\end{tabular}

We term this is the "independent 3D HOSVD" or "3DIHOSVD." For 3D- IHOSVD, we calculate the patch similarity independently on the three channels gained later PCA with the distance threshold $\tau_{d}=3 \sigma^{2} \mathrm{n}^{2}$ whereas in LJSCPW [11], the patch similarity is calculated merely over the $\mathrm{Y}$ channel from the $\mathrm{YCbCr}$ color space, which ignores chrominance information. For 4D-HOSVD/4DHOSVD2, the distance threshold used was $\tau_{d}=3 \times$ $3 \sigma^{2} n^{2}$.The patch-size used for all algorithms was $8 \times 8$

\section{CONCLUSION}

The 4 D-HOSVD methods clearly outperformed NL Means, 3D-IHOSVD and LJSCPW. Its PSNR values are very slightly higher than those of LJSCPW; however, it 'out- performed LJSCPW on some images. Over and above PSNR comparisons, we have observed that at higher noise levels, 3D-IHOSVD and LJSCPW produce color artifacts that alter the hue, unlike 4D-HOSVD/4D-HOSVD2. Thus, 4DHOSVD2 is clearly a state-of-the-art denoising algorithm. We compare the performance of existing James-Stein type CPW $\mathrm{p}_{\mathrm{l}}^{\mathrm{LJS}}$ under the classic NLM frame with the 4 D-HOSVD. In particular, we set the search region $\mathbb{S}$ to $31 \times 31$ square, use a $15 \times 15$ Bcentred on the local pixel, and test performance for $3 \times 3,5 \times 5$ and $7 \times 7$ patches $\mathbb{P}$ respectively. Test images 1 are gray-scale with additive Gaussian noises of $\sigma \in$ $\{10,20,40,60\}$ We denoise each test image by using 200 temperature parameters ranging from $1 \%$ to $200 \%$ of $\sigma^{2}|\mathbb{P}|$. We see that the superiority of LJSCPW decreases as the noise level increases. This occurs because as increases, the optimal shrinkage coefficient $\mathcal{P}$ decreases. However, a 
smaller $\mathcal{P}$ in our shrinkage model (19) implies less difference between $\hat{\mathrm{x}}_{1}^{\mathrm{LSS}}$ and $\widehat{\mathrm{z}}_{\mathrm{l}}$ where $\widehat{\mathrm{z}}_{\mathrm{l}}$ is simply the denoising result of the zero CPW. This phenomenon is more salient when $\widehat{\mathrm{Z}_{1}}$ is already a very good estimator, However, when is not that good, LJSCPW shows noticeable improvement by using noisy data. It is clear that HOSVD helps keep image details and weak edges, and has a more random-like method noise than LJSCPW. The denoising performance is then evaluated by computing its mean and standard deviation in terms of PSNR [8], [9] and SSIM [11].

\section{REFERENCES}

[1] Buades, B. Coll, and J. Morel, "A review of image denoising algorithms, with a new one," Multiscale Model. Simul., vol. 4, no. 2, pp. 490-530, 2005.

[2] Buades, B. Coll, and J.-M. Morel, "A non-local algorithm for image denoises," in IEEE Compu. Soc. Conf. Computer Vision and Pattern Recognition, Jun. 2005, vol. 2, pp. 60-65, vol. 2.

[3] K. Dabov, A. Foi, V. Katkovnik, and K. Egiazarian, "Image denoising by sparse 3-d transform-domain collaborative filtering," IEEE Trans. Image Process., vol. 16 , no. 8, pp. 2080-2095, 2007

[4] Deledalle, V. Duval, and J. Salmon, "Non-local methods with sha- peadaptive patches (nlm-sap)," J. Math. Imag. Vis., vol. 43, no. 2, pp. 103-120, 2012.

[5] V. Duval, J. Aujol, and Y. Gousseau, "A bias-variance approach for the nonlocal means," SIAM J. Imag. Sci., vol. 4, no. 2, pp. 760-788, 2011.

[6] J. Salmon, "On two parameters for denoising with nonlocal means," IEEE Signal Process. Lett., vol. 17, no. 3, pp. 269-272, Mar. 2010.

[7] J. Darbon, A. Cunha, T. Chan, S. Osher, and G. Jensen, "Fast non- local filtering applied to electron cryomicroscopy," in IEEE Int. Symp. Biomedical Imaging: From Nano to Macro, 2008, pp. 1331-1334.
[8] R. Vignesh, B. T. Oh, and C.-C. Kuo, "Fast non-local means (nlm) com- putation with probabilistic early termination," IEEE Signal Process. Lett. vol. 17, no. 3, pp. 277-280, Mar. 2010.

[9] W. Zeng and X. Lu, "Region-based non-local means algorithm for noise removal," Electron. Lett. vol. 47, no. 20, pp. 1125-1127, 2011.

[10] W. James and C. Stein, "Estimation with quadratic loss," in Proc. Fourth Berkeley Symp. Mathematical Statistics and Probability, 1961, vol. 1, no. 36, pp. 1-379.

[11] Z. Wang, A. Bovik, H. Sheikh, and E. Simoncelli, "Image quality assessment: From error visibility to structural similarity," IEEE Trans. Image Process., vol. 13, no. 4, pp. 600-612, 2004.

[12] L. de Lathauwer, "Signal Processing Based on Multilinear Algebra," PhD dissertation, KatholiekeUniversiteit Leuven, Belgium, 1997

[13] Donoho and I. Johnstone, "Ideal Spatial Adaptation by Wavelet Shrinkage," Biometrika, vol. 81, pp. 425-455, 1993.

[14] L. Zhang, W. Dong, D. Zhang and G. Shi, "Two-Stage Image Denoising by Principal Component Analysis with Local Pixel Grouping," Pattern Recognition, vol. 43, no. 4, pp. 1531-1549, 2010.

[15] AjitRajwade, AnandRangarajan and Arunava Banerjee, "Image Denoising Using the Higher Order Singular Value Decomposition" IEEE Transactions On Pattern Analysis and Machine Intelligence, Vol. 35, NO. 4, APRIL 2013

[16] Yue Wu, Brian Tracey, PremkumarNatarajan, and Joseph P. Noonan James-Stein Type Center Pixel Weights for Non-Local Means Image Denoising IEEE Signal Processing Letters, Vol. 20, No. 4, April 2013. 